\title{
Study of Oenological Fermentation: Which Strategy and Which Tools?
}

\author{
Jean-Roch Mouret ${ }^{1}$, Evelyne Aguera ${ }^{2}$, Marc Perez ${ }^{1}$, Vincent Farines ${ }^{1}$ and Jean-Marie Sablayrolles ${ }^{1, *(D)}$ \\ 1 SPO, INRAE, Institute Agro, University of Montpellier, 34000 Montpellier, France; \\ jean-roch.mouret@inrae.fr (J.-R.M.); marc.perez@inrae.fr (M.P.); vincent.farines@inrae.fr (V.F.) \\ 2 Pech Rouge, INRAE, 11430 Gruissan, France; evelyne.aguera@inrae.fr \\ * Correspondence: jean-marie.sablayrolles@inrae.fr; Tel.: +33-4-9961-2500
}

Citation: Mouret, J.-R.; Aguera, E.; Perez, M.; Farines, V.; Sablayrolles, J.-M. Study of Oenological Fermentation: Which Strategy and Which Tools?. Fermentation 2021, 7, 155. https://doi.org/10.3390/ fermentation7030155

Academic Editor: Antonio Morata

Received: 28 July 2021

Accepted: 12 August 2021

Published: 16 August 2021

Publisher's Note: MDPI stays neutral with regard to jurisdictional claims in published maps and institutional affiliations.

\begin{abstract}
Wine fermentation is a specific and complex research subject and its control is essential to ensure full process completion while improving wine quality. It displays several specificities, in particular, (i) musts with a very high sugar content, low $\mathrm{pH}$, and some limiting nutrients, as well as a great variability in must composition according to the year, grape variety, and so on; (ii) atypical fermentation conditions with non-isothermal temperature profiles, a quasi-anaerobiosis and legal constraints with a limited and predefined list of authorized operations. New challenges have emerged, related to the increasing diversity of commercially available yeast strains; the fluctuating composition of musts, particularly owing to climate change; and sustainability, which has become a key issue. This paper synthesizes approaches implemented to address all these issues. It details the example of our laboratory that, for many years, has been developing an integrated approach to study yeast diversity, understand their metabolism, and develop new fermentation control strategies. This approach requires the development of specific fermentation devices to study yeast metabolism in a controlled environment that mimics practical conditions and to develop original fermentation control strategies. All these tools are described here, together with their role in the overall scientific strategy and complementary approaches in the literature.
\end{abstract}

Keywords: wine; fermentation; study; experimental devices; tools; strategy

\section{Introduction}

Alcoholic fermentation is a key step in winemaking. During this process, hexoses and other grape must constituents are converted to ethanol, carbon dioxide, and many other secondary by-products that affect the sensorial properties of wine. Yeast strains have a major effect on the process, but must composition and the way the fermentation is controlled are also essential, so that the course of fermentation always depends on interactions between these three factors. This explains why alcoholic fermentation under oenological conditions is a specific and complex research subject.

The yeast strains used belong mainly to the $S$. cerevisiae species, but their number and diversity are constantly increasing, and increasingly more attention is being paid today to non-Saccharomyces species, mixed cultures, and natural microbial ecosystems.

Grape juice is characterized by a very high sugar content, a very low $\mathrm{pH}$, a quasianaerobiosis, and low concentrations of certain nutrients, notably assimilable nitrogen. During fermentation, this leads to a succession of deficiencies and stresses that result, in particular, in (i) a long stationary phase during which a large part of the sugars is metabolized in the absence of yeast growth and (ii) significant risks of mortality by the end of fermentation.

Winemaking fermentation control is essential to ensure a successful completion of the process, but also to limit energy costs and to improve the wine organoleptic characteristics. Certain specificities are to be considered, in particular temperature profiles, which are often non-isothermal owing to the strong exothermic potential of fermentation, and 
oxygen additions, usually necessary for yeasts, but that must be very low (a few mg/L) to avoid detrimental effects on quality. Legal constraints must also be considered, as only a limited number of operations are authorized, mainly in terms of nutrient additions and temperature control.

Much progress has been made in the last few years, but, in parallel, new challenges have emerged. Indeed, available yeast strains are more and more numerous and diverse; the composition of musts has evolved considerably (in particular because of climate change); and winemaking sustainability has become an essential issue, with, for example, a reduction in input quantity and the prospect of vinifying new grape varieties, in particular those resistant to downy and powdery mildews.

This paper synthesizes approaches implemented to address all these issues. It details the example of the research work of our laboratory that has, for many years, been developing an integrated approach to study yeast diversity, understand their metabolism, and develop new fermentation control strategies. This approach implies the use of specific setups, most of which have been custom-developed, to study yeasts in a controlled environment that mimics practical conditions and to implement innovative fermentation controls. These tools are described as well as their role in the overall scientific strategy, with one of the main questions being, "which setup for which research question?" Complementary approaches from the literature are also discussed.

\section{Study of Metabolism}

\subsection{Online Fermentation Monitoring}

\subsubsection{Development of Online Fermentation Monitoring Devices}

The study of yeast metabolism requires the monitoring of fermentation process dynamics. To improve this monitoring, we focused very early on the online monitoring of the main reaction, i.e., the production of ethanol and $\mathrm{CO}_{2}$ [1]. The method we chose, also proposed in other laboratories [2], was the measurement of $\mathrm{CO}_{2}$ release, with adequate frequency and accuracy in order to calculate in real time the instantaneous rate of fermentation. This laboratory setup ( $36 \times 1 \mathrm{~L}$ fermenters) was then extended to both pilot scale $(16 \times 100 \mathrm{~L}$ tanks) and small scale, with a fermentation robot $(360 \times 20 \mathrm{~mL}$ or $90 \times 300 \mathrm{~mL})$ (Figure 1).

The next step was to monitor online the synthesis of some molecules of interest, mainly the fermentative aromas (higher alcohols and esters) and main sulfur compounds [3,4]. The chosen technique was the use of online GC, with automatic sampling in the gas phase (sampling frequency $=1 \mathrm{~h}$ ). Today, two devices are operational: (i) at the $10 \mathrm{~L}$ and $100 \mathrm{~L}$ scales, a GC-MS-FPD to analyze several dozens of (mainly carbonaceous) compounds; and (ii) at laboratory scale (2 L), a compact GC-FID-PFPD particularly well adapted to the analysis of the main sulfur compounds. In additional studies on gas-liquid equilibrium, we modeled the partition coefficient $\left(\mathrm{k}_{\mathrm{i}}\right)$ for the main compounds [5-7]. For each of these compounds, it then became possible to compute real-time balances between the quantity accumulated in the liquid and that lost in the gas (Figure 2). The sum of both fractions corresponds to the actual quantity produced by yeast, which is the parameter with the highest metabolic interest.

\subsubsection{Interest in Online Fermentation Monitoring}

Thanks to online monitoring, with dozens or hundreds of measurements during fermentation, new information of direct interest for the study of metabolism is now available:

A. The dynamics of the synthesis of different compounds and their timing, with, sometimes, the highlighting of peaks that would have gone undetected with a manual sampling;

B. The calculation of the production rates of $\mathrm{CO}_{2}$ and various metabolites. These rates, which can be transformed into specific production rates if the cell population is also monitored, are directly proportional to metabolic fluxes. By combining the 
monitoring of several compounds, it is also possible to calculate the evolution of production yields, which represent further information of metabolic interest.
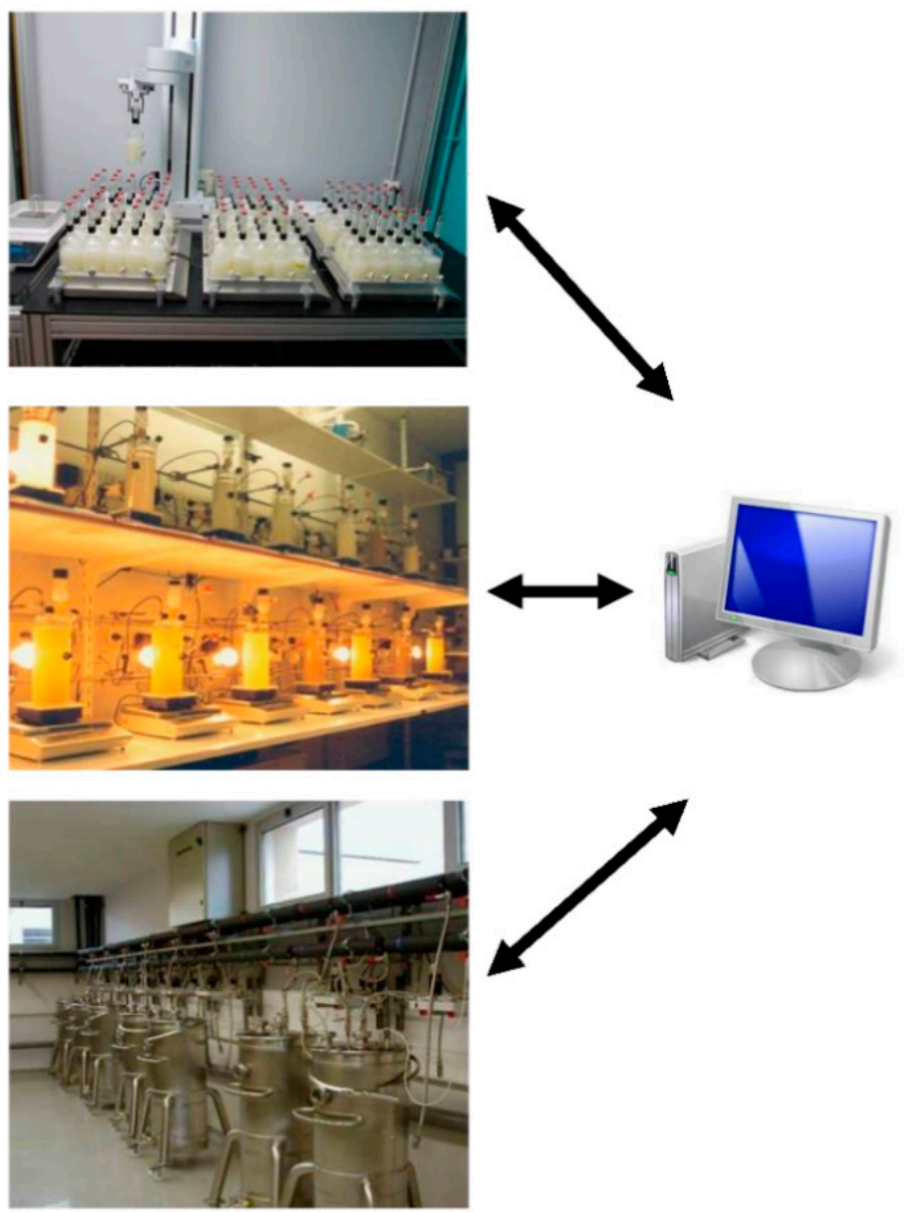

Figure 1. Online monitoring of $\mathrm{CO}_{2}$ production rate at laboratory and pilot scale. Fermentation robot $(360 \times 20 \mathrm{~mL}$ or $90 \times 300 \mathrm{~mL})$, laboratory setup $(16 \times 1 \mathrm{~L}$ fermenters $)$, and pilot scale $(16 \times 100 \mathrm{~L}$ tanks).

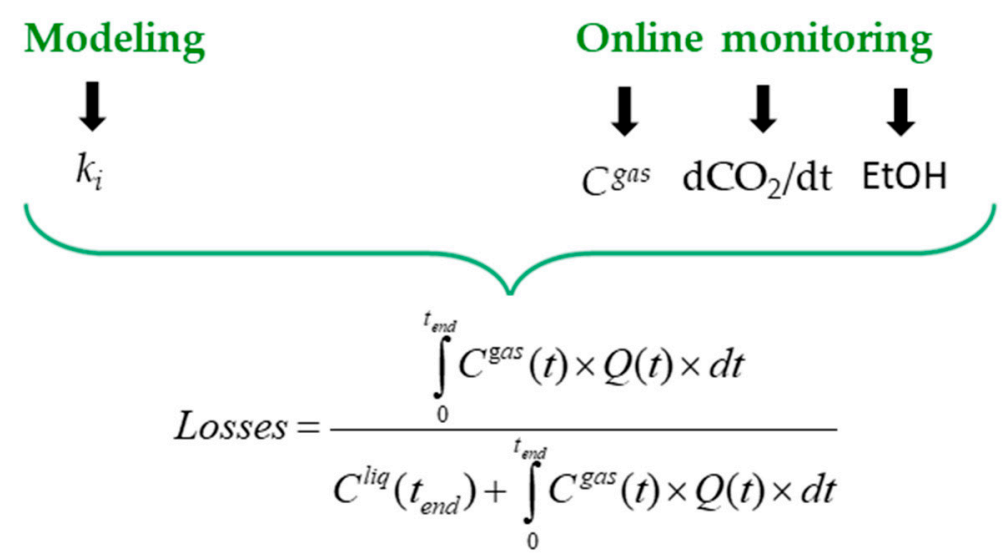

Figure 2. Calculation of the overall losses of a volatile compound in the exhaust gas from (i) online monitoring of the fermentation kinetics $\left(\mathrm{dCO}_{2} / \mathrm{dt}\right.$ and $\left.\mathrm{EtOH}\right)$ and concentration of the volatile compound in the gas phase ( $\left.\mathrm{C}^{\text {gas }}\right)$ and (ii) the modelling of the gas-liquid equilibrium coefficient $\left(\mathrm{k}_{\mathrm{i}}\right) . \mathrm{Q}(\mathrm{t})$ is the $\mathrm{CO}_{2}$ production rate (per liter of must) and $\mathrm{C}^{\mathrm{liq}}(\mathrm{t})$ the concentration of the volatile compound in the liquid phase [6]. 
It is then possible to couple these data with approaches such as metabolic flux analysis or transcriptomics. For example, this was achieved [8] when studying the effect of sterol additions during fermentation. These authors highlighted an increase in production yield between isoamyl alcohol and isoamyl acetate (Figure 3), in such a way that they could precisely define the sampling times before and after this yield change (slope change) to perform transcriptomic analyses on yeast cells in two distinct, well-defined physiological states.

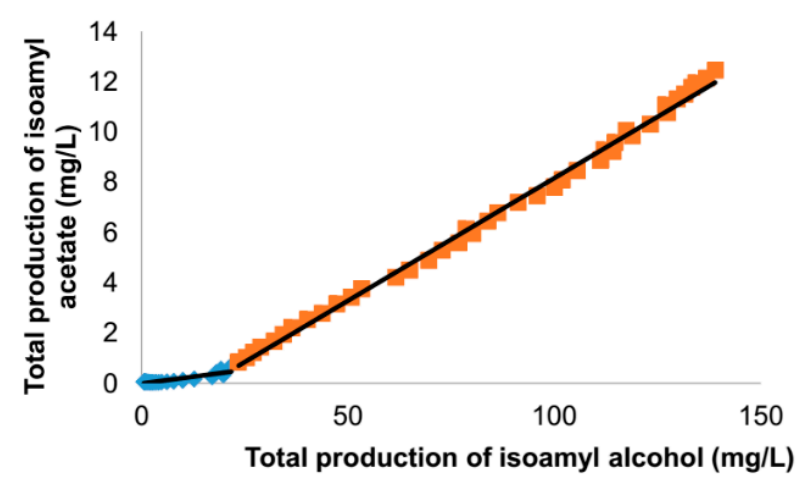

Figure 3. Online GC monitoring of fermentation aromas in $100 \mathrm{~L}$ tanks. Focus on the bioconversion of isoamyl alcohol to isoamyl acetate. Changes in total isoamyl acetate production as a function of total isoamyl alcohol production. The total productions represent the sum of the concentration in the liquid and the amount lost in the gas phase.

For volatile compounds, real-time balances between the gas and liquid phases enable differentiation between microbiological and physical phenomena. Thus, for example, when comparing fermentations at $20{ }^{\circ} \mathrm{C}$ and $30{ }^{\circ} \mathrm{C}$ [5], Morakul et al., 2011 calculated that the total production of ethyl hexanoate was $1.61 \mathrm{mg} / \mathrm{L}$ and $1.22 \mathrm{mg} / \mathrm{L}$, respectively, whereas the final concentrations in the wines were $0.92 \mathrm{mg} / \mathrm{L}$ and $0.36 \mathrm{mg} / \mathrm{L}$, respectively. This example shows that (i) the quantity of ester lost can be very high, in this case, $43 \%$ at $20{ }^{\circ} \mathrm{C}$ and $70 \%$ at $30{ }^{\circ} \mathrm{C}$; and (ii) the temperature effect is very different depending on whether one considers the total production (which reflects yeast metabolic capability) or the concentration in the wine, with $30 \%$ and $150 \%$ increases between $30{ }^{\circ} \mathrm{C}$ and $20{ }^{\circ} \mathrm{C}$, respectively. If considering only the liquid content of ester, the temperature impact on yeast metabolism is highly overestimated.

Thus, integrated approaches can be implemented, such as the one carried out on the production of fermentation aromas, which combines online monitoring with biochemical engineering and systems biology approaches (Figure 4).

\subsubsection{Interest of Industrial Sensors}

In addition to these specific devices, we considered whether using industrial sensors was relevant in the context of monitoring oenological fermentation.

The value of measuring medium conductivity has been demonstrated [9]. Indeed, its evolution is very well correlated to the variation of assimilable nitrogen concentration. This measurement is thus very relevant to follow in a very fine way the dynamics of nitrogen assimilation at the beginning of fermentation and after additions of nitrogenous nutrients.

The measurement of dissolved $\mathrm{CO}_{2}$ is very useful for monitoring very precisely the recovery of yeast activity at the very beginning of fermentation [10]. It has also been used to study the rehydration phase of active dry yeasts [11].

Monitoring dissolved oxygen has made it possible to calibrate the oxygenation system [12]. Monitoring during fermentation is of little interest because the oxygen content is almost always equal to zero, even during the oxygenation phases, as the rate of consumption by yeast is generally higher than the supply rate. However, recent sensors allow more practical analyses and make this measurement potentially interesting to follow, for example, the heterogeneity within large volume tanks [13]. 


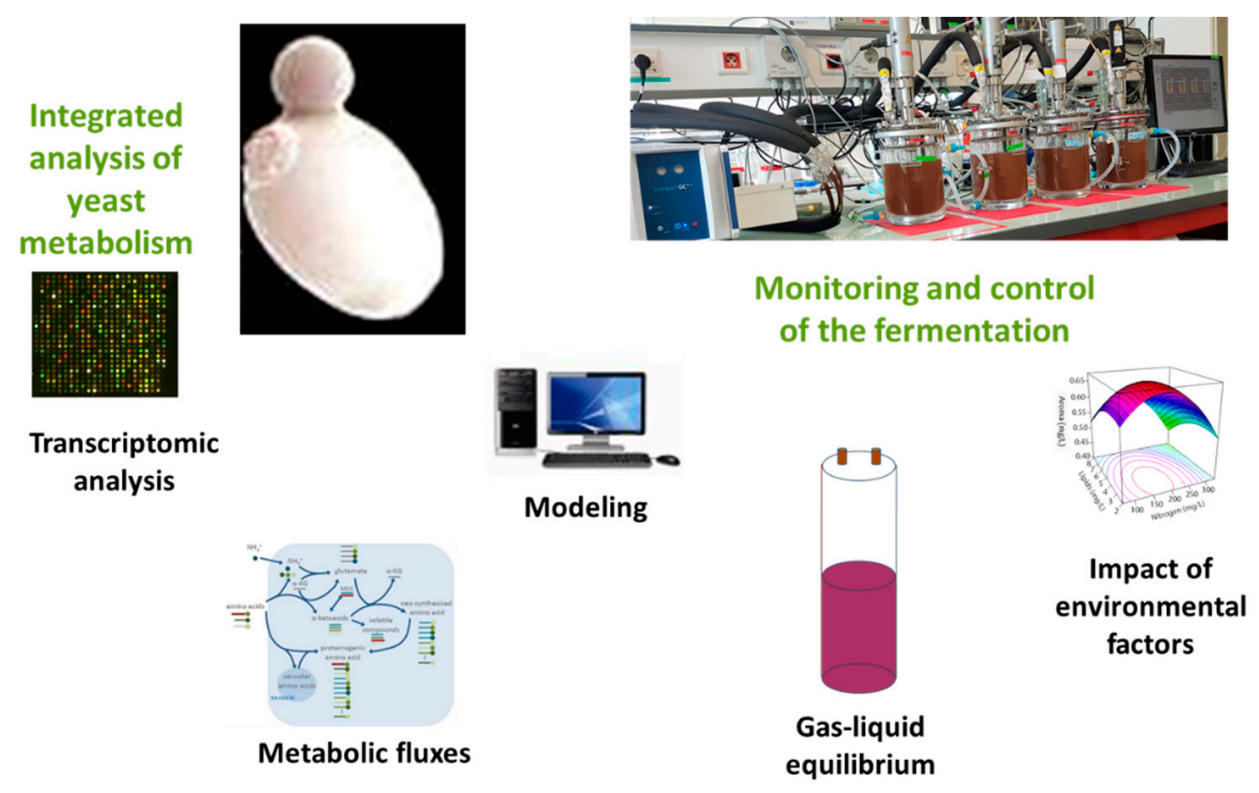

Figure 4. Integrated approach combining biochemical engineering and system biology. Example of fermentative aroma synthesis. Online monitoring and fermentation control tools make it possible to (i) carry out cultures under perfectly controlled conditions; (ii) monitor parameters of metabolic interest: fermentation rate, and synthesis dynamics of the main fermentative aromas (with total production, i.e., concentration in the liquid + losses); (iii) cross-reference this information with system biology approaches: choice of key moments for post-genomic analyses and comparison of metabolic flux estimates (production rates vs. flux modelling).

The evolution of redox potential and $\mathrm{pH}$ have also been monitored. They highlighted the main phases of fermentation [14]. However, it is difficult to obtain more precise information because these values are influenced by many compounds, and thus vary in relation to must composition.

The above results were obtained using fermentors or tanks. Other authors have also shown the benefit of combining different industrial sensors (temperature, humidity, level) to monitor the evolution of vinifications in barrels [15].

\subsection{Design of Dedicated Fermentation Tools}

In addition to online monitoring systems, we developed several fermentation tools dedicated to the study of yeast metabolism under oenological conditions.

\subsubsection{Controlled Rate Fermentations}

The $\mathrm{CO}_{2}$ production rate, which is proportional to yeast activity, can be regulated by real-time temperature control or the supply of nutrients, mainly assimilable nitrogen. This possibility has been very useful, especially for studying the nitrogen requirements of different yeast strains by comparing the nitrogen inputs necessary to achieve fermentations at identical rates [16,17].

\subsubsection{Controlled Micro-Oxygenation}

The addition of small amounts of oxygen (a few $\mathrm{mg} / \mathrm{L}$ ) is very useful for the completion of fermentation, but the management and quantification of this oxygenation are often poorly controlled, even at the laboratory scale. To address this issue, we developed a device to control micro-oxygenation during fermentation [12]. Oxygen is transferred by diffusion through a silicone tube and the amount transferred can be controlled, via the supervision software, either as a function of time or as a function of the reaction progress (e.g., $1 \mathrm{mg} / \mathrm{L}$ per \% of ethanol produced). This device is very convenient to study the impact of oxygen not only on the main reaction, but also on the production of compounds 
such as fermentative aromas [18], sulfur compounds, acetaldehyde, diacetyl, and so on, as well as under rosé or red wine conditions, on color, and polyphenolic compounds (Figure 5).

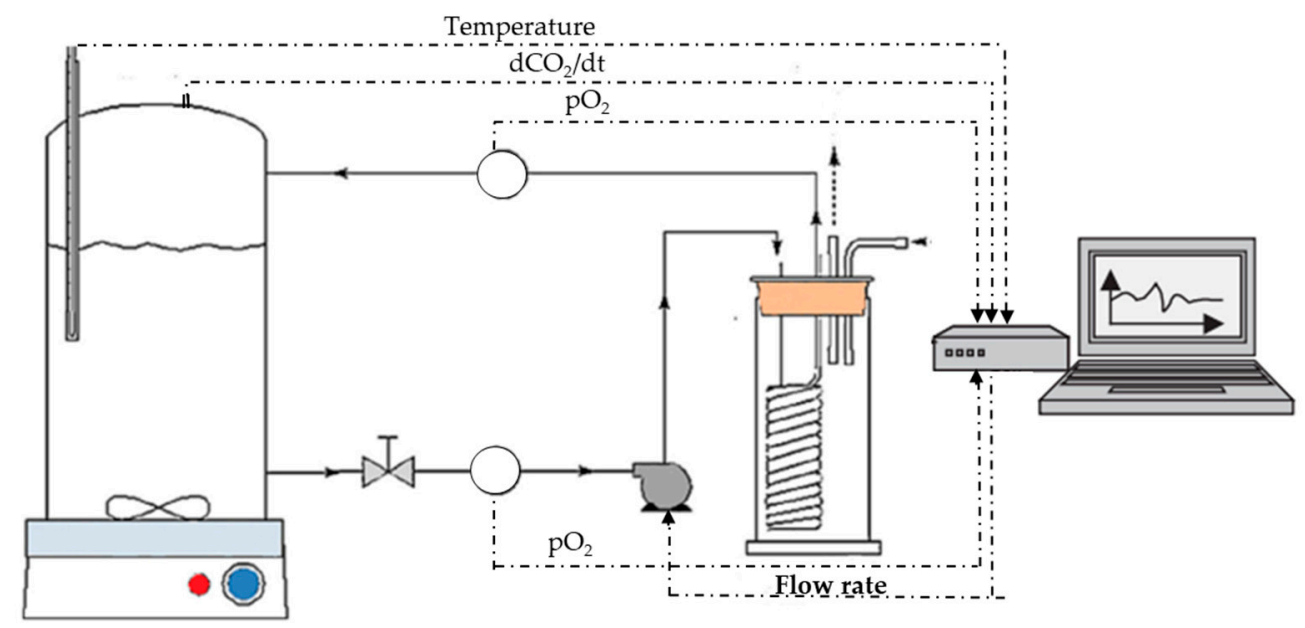

Figure 5. Schematic representation of the bubble-free micro-oxygenation system. Oxygen is added by diffusion across a silicone tubing membrane with a controlled oxygen transfer rate (OTR). The OTR is modulated by controlling the liquid flow rate inside the silicone tube.

It should be noted that this system dedicated to the control of oxygenation during fermentation (addition of a few $\mathrm{mg} / \mathrm{L}$ per hour or per day) is not adapted as it is to microoxygenation during wine aging, which requires additions of a few $\mathrm{mg} / \mathrm{L}$ per month [19].

\subsubsection{Multi-Stage Continuous Bioreactor}

In winemaking conditions, yeasts go through a growth phase and then a stationary phase, during which more than half of the sugars is fermented. A comprehensive study of wine yeast physiology must thus include yeasts in a non-growing phase. To obtain nongrowing yeasts in a steady state, we developed a four-stage continuous reactor that mimics, in a series of steady states, the conditions of batch fermentation (Figure 6) [20]. Despite its complexity, this fermentation device has shown its great interest for metabolic studies. For example, when studying valine uptake by yeast, it was shown that the corresponding transcriptomic data are of better quality than in a batch fermentation [21]. It can also be noted that, in addition to its interest in the study of metabolism, this multistage fermenter has been used, in collaboration with automaticians, as a model to develop control strategies for non-linear and constrained systems [22]. 


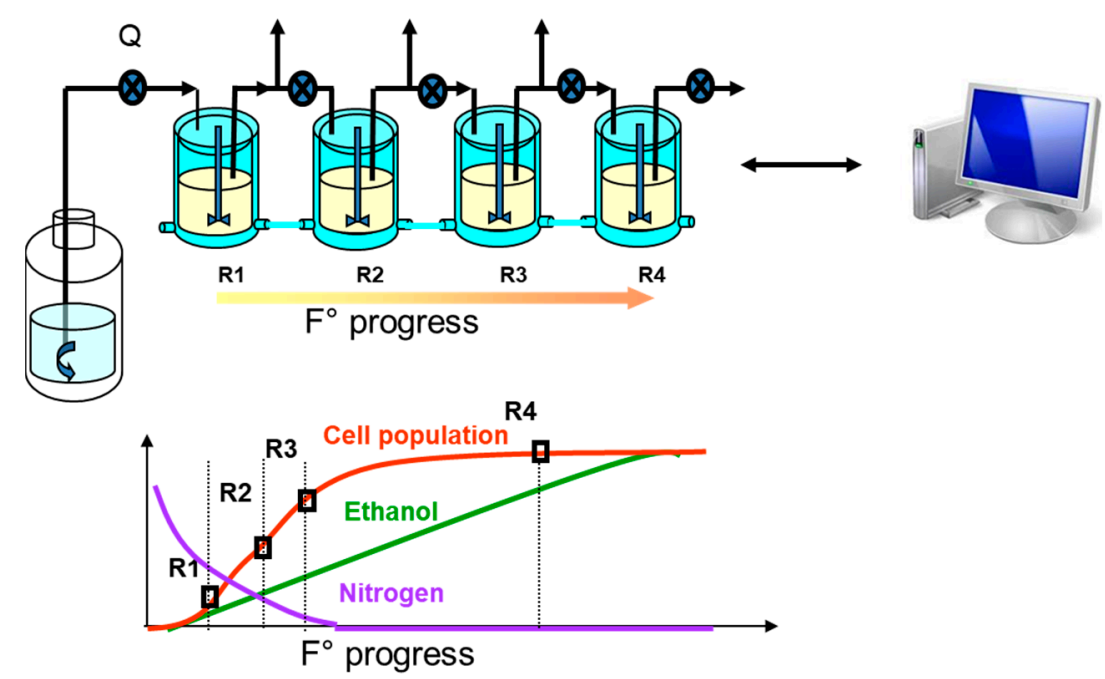

Figure 6. Schematic representation of the four-stage continuous fermentation device. The system is made up of four tanks (R1 to R4) linked in series. The five pumps are monitored independently by the control software, which also integrates the data coming from the mass flowmeters. Each bioreactor, except the first one, is fed with cells and partially fermented medium from the previous tank. The first stages are run with growing cells, while the last ones operate as resting cells. This device reproduces batch fermentation characteristics.

\section{Simulation of Industrial Scale}

An important issue is the simulation of industrial conditions in order to be as relevant as possible when studying yeast metabolism and fermentation control, and thus to use industrial-scale trials, which are generally very poorly controlled, only for the validation step.

\subsection{Non-Isothermal Temperature Profiles}

The usual laboratory or pilot scale facilities do not allow for fermentation under nonisothermal conditions comparable to those observed on a large scale. Indeed, in large tanks, outside the regulation phases, there are often temperature increases of up to $10^{\circ} \mathrm{C}$ or more, and these temperature profiles greatly modify fermentation kinetics. Our idea was thus to incorporate a thermal model into the control software that calculates the temperature evolution that would occur in an industrial tank $[1,23]$. This model can also be used to estimate the evolution of the energy demand for temperature control throughout the fermentation, regardless of the temperature profile [24].

\subsection{Hydrodynamic Conditions}

Hydrodynamic conditions in small fermenters differ from those in large tanks (several hundred hectoliters), particularly owing to solid particles and $\mathrm{CO}_{2}$ release, and this must be taken into account when extrapolating to different scales.

\subsubsection{White and Rose Winemaking}

Plouy, 2000 and Casalta et al., 2010 studied the effect of stirring and turbidity on white wine fermentation kinetics in $1 \mathrm{~L}$ and $100 \mathrm{~L}$ fermenters $[25,26]$. In particular, they concluded that, in small (laboratory-scale) fermenters, agitation has several advantages: (i) it reduces differences with the pilot scale and increases kinetics reproducibility, (ii) it avoids any decrease in the cell population in suspension at the end of fermentation, and (iii) it decreases the negative effect of excessive must clarification.

Malherbe, 2003 compared fermentation kinetics in $100 \mathrm{~L}$ and 11,000 L tanks and found them highly reproducible, indicating that kinetics of industrial tanks can be satisfactorily reproduced at the pilot scale [27]. However, additional experiments revealed slight dif- 
ferences, especially during sluggish fermentations, with very low fermentation rates at the end.

\subsubsection{Red Winemaking}

In red winemaking, maceration represents the major issue and industrial conditions cannot be perfectly reproduced on a small scale.

At the laboratory scale $(1.5 \mathrm{~L})$, we currently use 'Bodum' type fermenters with a good reproducibility of experiments and the possibility of studying a large number of conditions. The interest of these fermenters has been described by Dambergs and Sparrow [28].

However, the pilot scale $(100 \mathrm{~L})$ is much more appropriate to simulate industrial conditions and to understand the mechanisms involved, especially when using online kinetics monitoring. For example, Aguera and Sablayrolles, 2005a observed cap formation (Figure 7A) and the effect of cap punching on fermentation kinetics (Figure 7B) [29]. They pointed out a highly significant increase in $\mathrm{CO}_{2}$ production rates after cap punching. This increase was (i) nearly instantaneous, persisting over several hours, and (ii) higher when pumping over was carried out during the second part of the fermentation (stationary phase). Cell population measurements indicated that this kinetics acceleration was mostly the result of a transfer of yeasts from the cap to the liquid phase, increasing the size of the cell population in the liquid (by more than $50 \%$ ).

(A)

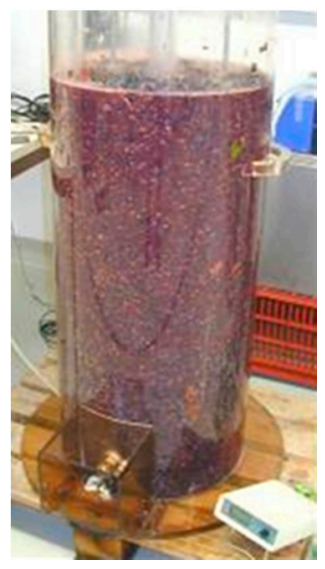

(a)

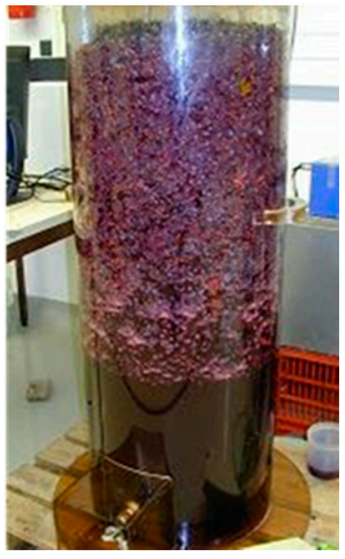

(b)

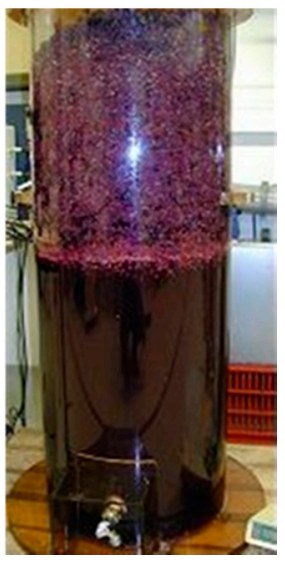

(c)

(B)



Figure 7. (A) Cap formation during red wine fermentation. (a) Tank filling, (b) after fermentation of $5 \%$ of the sugar, (c) after fermentation of $40 \%$ of the sugar. (B) Effect of cap punching on fermentation kinetics. Increase in $\mathrm{CO}_{2}$ production rates after cap punching owing to a transfer of yeasts from the cap to the liquid phase, increasing the size of the cell population in the liquid.

The possibity of miniaturizing red wine vinification to a pilot or even laboratory scale has been discussed by several authors. Schmid et al., 2009 measured the same temperature gradients between the cap and liquid phase in $50 \mathrm{~L}$ and $35 \mathrm{hL}$ tanks [30]. Sparrow et al., 2015 
and Sampaio et al., 2007 observed good consistency in phenolic composition at different scales, while Lopes et al., 2002 were able to predict the evolution of indigeneous populations in industrial tanks from laboratory fermentations [31-33]. However, by studying coinoculations, Tufariello et al., 2020 obtained quite distinct fermentative aroma production according to scales [34].

\subsection{Wine Quality}

It is essential to consider the organoleptic quality of the wine at least during the last steps of the research programs. This quality can be dependent on the scale of production. Moreover, it is very difficult to estimate at a very small scale even if the online or offline analysis of some marker molecules allows a first level of characterization.

The pilot scale is, once again, particularly relevant because the volume $(100 \mathrm{~L})$ is a good compromise for an expert jury to carry out a sensory analysis while testing numerous conditions, with the possibility of replicates. Moreover, the wine characteristics are close to those obtained on an industrial scale. This pilot scale can thus be used, for example, to validate the organoleptic value of new strains before their commercialization [35].

\section{Development of New Strategies for Fermentation Control}

Wineries are increasingly better equipped to control winemaking, especially alcoholic fermentation, with, for example, more and more precise temperature controls. On the other hand, control strategies still remain relatively empirical and are generally decided a priori, without taking into account must composition variability.

The rate of $\mathrm{CO}_{2}$ release is proportional to yeast activity (cf. part 1.1.2). It is also directly correlated to the rate of heat production and, therefore, to the amount of energy required to regulate the temperature [23]. Thus, this parameter is of major interest for the development of new fermentation control strategies optimized for each tank. The availability of online data on quality markers, mainly fruity aromas, also makes it possible to take into account quality-related parameters, even if sensory analysis cannot be replaced in the final validation steps.

Fermentation operations, mainly nutrient additions and temperature regulation, can be adapted to actual fermentation behavior, with the possibility of taking into account must composition variability, depending on the tank (cf. part 3.1). The huge amount of data on fermentation dynamics is also very useful to build and validate predictive models that can initially be used for simulation (cf. part 3.2) and later on for optimized control (cf. part 3.3).

\subsection{Individual Tank Control}

\subsubsection{Nutrient Addition}

The addition of nutrients is one of the main ways to control fermentation, but the challenge is to perform these additions only if they are necessary and in an optimized way.

With assimilable nitrogen generally being the limiting nutrient, Bely et al., 1990 proposed a method to (i) estimate its concentration in the must, based on the value of the maximum fermentation rate, and (ii) thus detect deficient musts to which nitrogen addition is recommended [36]. Their work also highlighted the effectiveness of additions made during the stationary phase. To complement this work, we subsequently tested controlled continuous additions and compared different sources of nitrogen to evaluate their impact on fermentation kinetics and the aromatic characteristics of wines [17,37]. The results could be compared to those obtained by other research groups, synthesized by Gobert et al., 2019 [38].

The positive effect of oxygenation has also been widely described, notably to limit the risks of stuck fermentations [39]. Online monitoring has allowed both (i) to determine the best timing of oxygen addition and (ii) to show that oxygenation has mainly an impact on the final fermentation kinetics. It thus dramatically differs from the effect of nitrogen additions. 
More recently, special attention has been given to the management of lipid compounds, especially in highly clarified musts [40-42]. In this specific case, turbidity has to be managed in the interaction with nitrogen and oxygen additions. Online kinetics monitoring is a very useful tool for differentiating the type of deficiency and to optimize corrections [43].

\subsubsection{Temperature Control}

Temperature profiles based on the control of fermentation rate and optimizing both the duration of fermentation and the energy required to regulate temperature were first proposed [44]. Later, the online monitoring of fermentative aromas provided a better understanding of the effect of temperature on both the synthesis of these molecules and their loss $[4,45]$. The importance of temperature control to fine-tune wine quality was also pointed out by Molina et al., 2007 [46].

\subsection{Modeling}

Several models have been developed. They are both (i) mechanistic, to consider the main biological or physico-chemical mechanisms involved, and thus widen the validity domain; and (ii) dynamic, to describe the evolution of the process monitored online.

The fermentation kinetics model calculates the evolution of the fermentation rate based on the initial concentration of available nitrogen and the temperature profile [47]. After being validated in a wide range of oenological situations, it was combined with the thermal model (cf. part 2.1) and introduced into a simulation and optimization software for alcoholic fermentation [24].

A dynamic model has been developed to predict the synthesis kinetics for the main aroma compounds produced by yeast during fermentation [48]. This model is a proof of concept and its coefficients' values may change when using different natural musts and yeast strains. However, the general structure of the model should remain valid regardless of the medium.

In parallel, the gas-liquid equilibrium of the main aromas was modeled for real-time calculation of concentrations in the gas and liquid phases as well as cumulative losses during the process $[4,5]$.

The four models can be combined (Figure 8) into an overall model to search for new predictive control and fermentation optimization strategies (cf. part 3.3) [49].

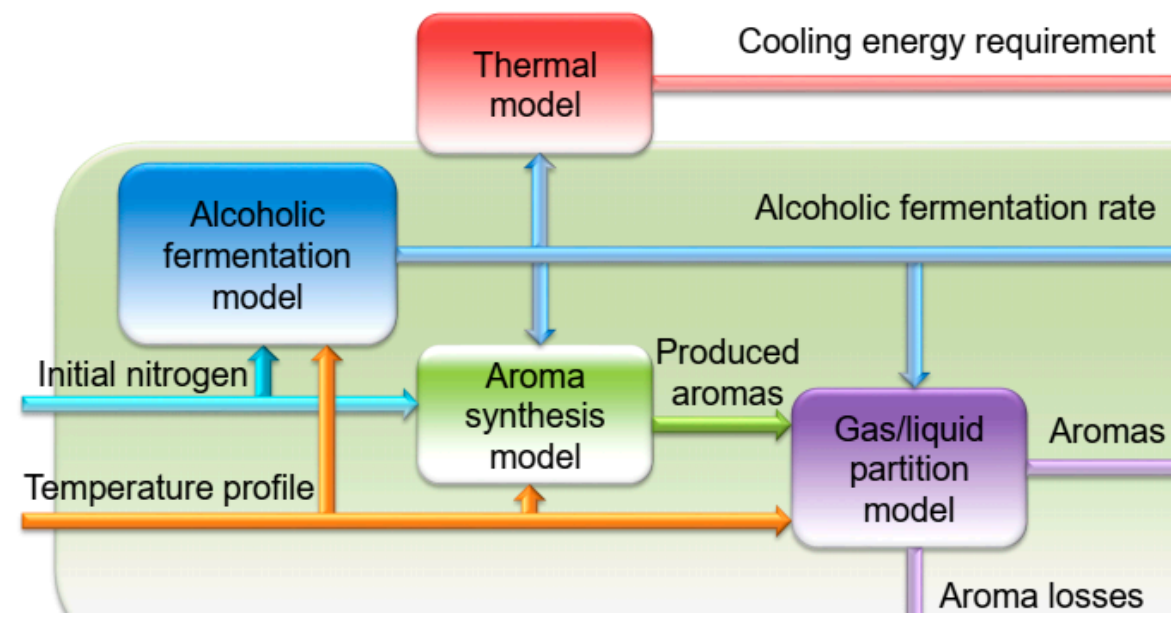

Figure 8. Structure of the global wine fermentation model. The global model integrates four models calculating the fermentation rate, the energy required for temperature regulation, the synthesis of the main fermentation aromas, and the distribution of these aromas between the gas and liquid phases.

\subsection{Predictive Control and Optimization}

A major challenge is to use modeling to anticipate and optimize the main fermentation parameters. The overall model takes into account both technological parameters and 
qualitative criteria and the optimization necessarily requires trade-offs. For example, low temperatures favor the synthesis of esters and limit their losses, but increase the fermentation duration. It is thus necessary to find a compromise in temperature management. The same holds true for the management of nutrient additions.

Obtaining wines with predefined aromatic profiles while optimizing tank use and energy expenses becomes a complex, but feasible issue in the framework of multidisciplinary collaborative projects including specialists in modeling, control, and optimization of bioprocesses.

In a first approach, Mouret et al., 2019 proposed a multi-criteria optimization in two phases: (i) generation of a Pareto front (set of optimal solutions) from the model, and then (ii) an interactive exploration (man-machine interface) of the Pareto front using a visualization software in order to progressively limit the number of possible solutions and ultimately define the optimal solution satisfying all the criteria [50].

A current project consists of coupling optimization and online monitoring of kinetics and aromatic compounds in order to develop a real-time control strategy. This is still a proof of concept with synthetic media and a limited number of marker molecules, but its application in real conditions, for the production of wines with well-defined aromatic profiles, can already be considered.

\section{Knowledge Management System}

Online data were also used to build a winemaking fermentation knowledge management system (ALFIS) [51]. In addition to remote monitoring, the objective was to manage and organize a large amount of data to extract key knowledge. The information system combines the acquisition of (i) online kinetics data from 52 fermenters (36 at lab scale and 16 at pilot scale) and the fermentation robot $(360 \times 20 \mathrm{~mL}$ or $90 \times 300 \mathrm{~mL}$ ); (ii) online GC data from six fermenters at pilot scale; (iii) offline data from biological analyses such as yeast cell number, cell size distribution (from the Coulter counter), and concentration of extracellular metabolites; and (iv) metadata (operation descriptions, faults, expert opinions, and so on). These data and metadata are formalized in XML and RDF, which provides flexibility and a generic method for managing heterogeneous, multisource data. ALFIS is available through a dedicated and secure web interface. Currently, it contains data resulting from more than 22,000 fermentations.

This big data management is particularly well adapted to the screening of a large number of strains or culture conditions. It is thus a valuable tool for current issues such as the study of biodiversity. ALFIS also permits the development of data analysis tools such as ontologies. For example, a specific ontology of events (faults or enological operations) was developed to automatically identify erroneous online measurements and avoid misinterpretation of fermentation data [51].

\section{Which Experimental Set-Up for Which Question?}

The choice of the experimental setup is essential to best answer research questions. For our equipment, the main issues are the number of fermentations, the scale, and the instrumentation needed. Another essential parameter is the medium, in particular the choice between a synthetic and a natural must.

\subsection{Equipment}

Figure 9 summarizes the main wine fermentation-related research questions and the most appropriate equipment in each case. 


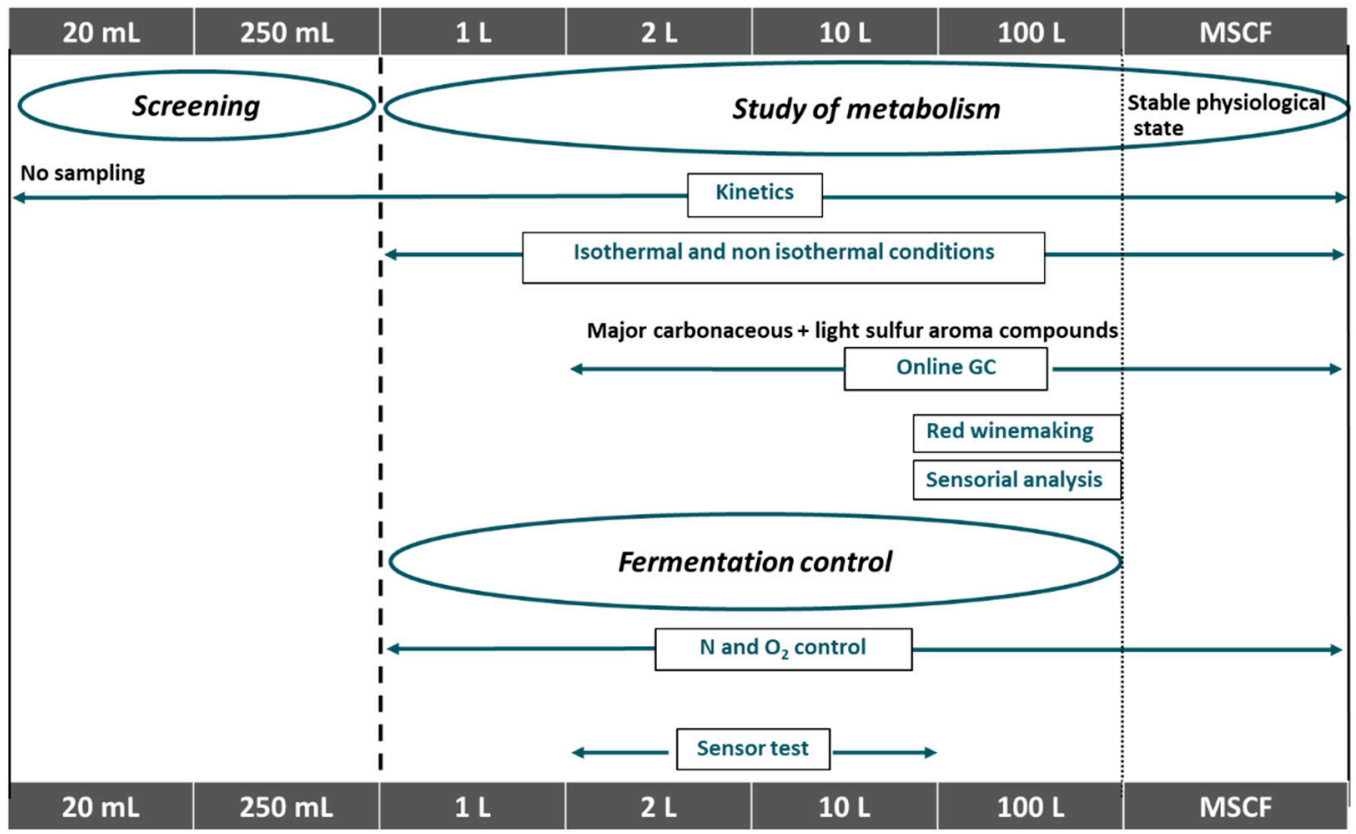

Figure 9. Which equipment for which research question? Choice of the scale (from $20 \mathrm{~mL}$ to $100 \mathrm{~L}$ ) and the equipment level (possibility of different online monitoring and control systems) according to (i) the research topic (screening, study of metabolism, or fermentation control) and (ii) specific needs (sensory analysis, red winemaking conditions, sensor test). MSCF: multistage continuous fermenter.

The fermentation robot is clearly dedicated to the screening of strains, media, and so on. When the number of conditions to be tested is very high (several hundreds, even thousands), the most appropriate scale is $20 \mathrm{~mL}$, with the possibility of testing up to 360 fermentations simultaneously. On the other hand, whenever multiple sampling during fermentation is required for offline analysis, only the $300 \mathrm{~mL}$ fermenters can be used, with no more than 90 fermentations running simultaneously.

Yeast metabolism can be studied at all scales from $1 \mathrm{~L}$ to $100 \mathrm{~L}$. The latter scale is mainly recommended to reproduce red wine making conditions or to perform a sensory analysis. The use of the multistage continuous fermenter (MSCF), whose implementation is more complex, is adequate when cells in a stable physiological state and/or in stationary phase are needed. The use of online GC is mainly dedicated to the analysis of carbonaceous (higher alcohols, esters, aldehydes, and so on) or sulfur $\left(\mathrm{H}_{2} \mathrm{~S}\right.$, mercaptans, and so on) fermentative aromas. The fermentation volume depends on the number of molecules to be studied, as there are more molecules available at the $10 \mathrm{~L}$ or $100 \mathrm{~L}$ scale with GC-MS.

Studying fermentation control is also feasible with fermenters from $1 \mathrm{~L}$ to $100 \mathrm{~L}$. Here, again, the $100 \mathrm{~L}$ scale is especially appropriate for red wine fermentation or for the implementation of sensory analysis. The controlled addition of micro quantities of oxygen (a few $\mathrm{mg} / \mathrm{L}$ ) is possible at all scales, but can only be controlled automatically at the $10 \mathrm{~L}$ and $100 \mathrm{~L}$ scales. As for the use of sensors, whether commercial, such as conductivity or redox sensors, or experimental, it is feasible at all scales, except in the case of specific constraints such as sensor size.

\subsection{Medium}

The choice of the culture medium is also essential according to the research issue.

Synthetic media are generally the most appropriate for metabolic studies because they have a perfectly controlled composition. In our laboratory, the reference medium is the one described by Bely et al., 1990, but it is often modified according to our needs [36]. Indeed, this medium simulates a must whose only limiting nutrient is nitrogen. This corresponds to most oenological situations. Nevertheless, the most common exceptions are lipid-deficient media, which corresponds, for example, to highly clarified musts [42]. In this last case, it is 
essential to modify the composition of the lipid fraction by varying its concentration and composition in sterols and fatty acids. This fraction is also of utmost importance in the study of the efficiency of oxygen additions, which is extremely different depending on the level of lipid deficiency in the medium $[17,40]$.

Sometimes, in particular projects, natural musts just cannot be substituted. This was the case, for example, in a study on stuck fermentations that required the collection of a large number of musts from diverse origins [39]. This is also the case for the validation of models and strains or for sensory analysis. The experimental cellar and the sterile storage of liquid phase musts are very useful to make available a variety of raw materials throughout the year. Small volumes $(10-20 \mathrm{~L})$ are stored in frozen form, while larger ones $(10-20 \mathrm{hL})$ are flash pasteurized before cold storage $\left(4^{\circ} \mathrm{C}\right)$ under inert gas [29]. This conservation has no impact on fermentation kinetics, provided that the addition of sludge is well managed [52]. Indeed, these solid particles sediment at a low temperature, resulting in very clear supernatants after several months of storage.

\section{Other Approaches: Prospects}

The tools will evolve according to the issues of the wine industry. Some of these evolutions are well identified, with specific approaches already being developed in different laboratories.

\subsection{High-Throughput Approaches}

Several questions, particularly those related to climate change, will require highthroughput approaches. Some of them will be at the viticulture-oenology interface, particularly in the context of varietal selection programs, where oenological parameters will have to be increasingly taken into account already from the first stages of selection.

High-throughput phenotyping tools are still often limited, especially for red winemaking fermentations. For this reason, we are currently involved in the development of an automated device for small volume $(<1 \mathrm{~kg})$ red wine fermentations. Compared with the current devices used in liquid-phase fermentation, the additional objective is to (i) control the maceration in a way that mimics industrial conditions as much as possible and (ii) monitor online the parameters related to polyphenolics' extraction.

\subsection{Mixed Cultures and Ecosystems}

Non-conventional yeasts are increasingly used to broaden the organoleptic characteristics of wines [53,54]. Non-Saccharomyces (NS) strains are usually unable to complete wine alcoholic fermentation. This limitation can be overcome through the use of mixed inoculations with S. cerevisiae, but controlling such cultures remains a difficult challenge [55]. Sequential inoculation, with delayed S. cerevisiae inoculation to allow for NS strain implantation, represents the best strategy; its fully successful implementation is complex and highly dependent on the strains used. In general, optimizing the management of NS/S mixed fermentations requires a better understanding of the interactions between strains, especially cell-to-cell mechanisms and the role of acetaldehyde [56].

Our laboratory has not developed any device dedicated to mixed cultures, but several have been described in the literature:

A. Double-compartment reactors with a physical separation of two yeast populations while culture medium homogeneity is maintained in both compartments $[57,58]$. These reactors, dedicated to research, are of great interest for studying interaction mechanisms between strains;

B. Immobilized yeasts bioreactors. There are several methods for yeast immobilization: the use of natural supports (e.g., fruit pieces), organic supports (e.g., alginate), inorganic supports (e.g., porous ceramics), membrane systems, and multifunctional agents $[59,60]$. However, these technologies, which have potential interest at both the research and application levels, are yet to be optimized [61,62]. 


\subsection{Sensors}

In addition to the possible evolution of the reactors, their instrumentation with new or more efficient sensors is also a potential area of evolution. The major challenge is no longer to monitor the main reaction, for which many sensors have already been proposed, even recently [63], but to obtain complementary information, mainly on yeasts and their physiological state and on the main molecules of interest (polyphenols, aromas, and so on).

Flow cytometry is undoubtedly the most promising method to simultaneously obtain the quantification of yeast cells; the determination of their viability; and the assessment of their physiology, e.g., by morphological analysis of the budding division process $[64,65]$. The latter authors carried out online monitoring on a laboratory scale, thanks to automated sampling and sample processing (dilution and addition of a fluorochrome), but they considered that this monitoring is currently difficult to implement for process control.

Several authors have developed sensors to monitor color evolution during maceration in red winemaking $[66,67]$. Voltammetric methods for the determination of wine polyphenolic compounds and total antioxidant capacity are under development, and electrochemical sensors may be expected to replace conventional methods for color and total polyphenols' measurements and the assessment of wine oxidation state [68]. All these measurements are of great interest under standardized and well-known conditions, but owing to the complexity of polyphenols' chemistry, it is still very difficult to evidence general relationships between this information and wine quality.

Different devices for online measurement of fermentation aromas have also been investigated. Electronic noses have been tested by several authors [69-72]. These devices are particularly interesting for the analysis of complex mixtures, but are still under development and cannot yet be used for online monitoring of wine aromas during fermentation.

Some techniques such as ATR-FTIR or FTICR-MS spectroscopy are sensitive to a very large number of compounds in the fermentation medium and can already be used to define different 'signatures' depending, for example, on the nitrogen source or the strain used in bioprotection $[73,74]$. The challenge is to progress in the analysis of this very rich, but also very complex information.

\section{Conclusions}

The study of oenological fermentation remains more than ever a major challenge because this key step in the winemaking process must now face important changes in the composition of grapes as well as a strong evolution of consumer expectations in terms of product quality and process sustainability.

The new research questions that arise concern both yeasts and the control of fermentation. This is why integrated approaches involving different disciplines (genetics, physiology, biochemical engineering) must be developed in order to propose effective and realistic solutions to the industry.

Author Contributions: Conceptualization, J.-R.M., E.A., M.P., V.F., J.-M.S.; writing-preparation of original version, J.-M.S.; writing-revision and editing, J.-R.M., E.A., M.P., V.F., J.-M.S. All authors have read and agreed to the published version of the manuscript.

Funding: This research received no external funding.

Institutional Review Board Statement: Not applicable.

Informed Consent Statement: Not applicable.

Data Availability Statement: No new data were created or analyzed in this study. Data sharing is not applicable to this article.

Acknowledgments: The authors would like to thank Christian Picou for his invaluable technical assistance and all colleagues from the SPO, Pech Rouge, MISTEA, and SAYFOOD research units who contributed to the development of the fermentation devices described in this article.

Conflicts of Interest: The authors declare no conflict of interest. 


\section{References}

1. Sablayrolles, J.M.; Barre, P.; Grenier, P. Design of a laboratory automatic system for studying alcoholic fermentation in anisothermal enological conditions. Biotechnol. Tech. 1987, 1, 181-184. [CrossRef]

2. Nerantzis, E.T.; Tataridis, P.; Sianoudis, I.A.; Ziani, X.; Tegou, E. Winemaking process engineering on line fermentation monitoring-Sensors and equipment. Sci. Technol. 2007, 5, 29-36.

3. Morakul, S.; Mouret, J.R.; Nicolle, P.; Aguera, E.; Sablayrolles, J.M.; Athes, V. A dynamic analysis of higher alcohol and ester release during winemaking fermentations. Food Bioprocess. Technol. 2013, 6, 818-827. [CrossRef]

4. Mouret, J.R.; Perez, M.; Angenieux, M.; Nicolle, P.; Farines, V.; Sablayrolles, J.M. On-line-based kinetic analysis of higher alcohol and ester synthesis during winemaking fermentations. Food Bioprocess. Technol. 2014, 7, 1235-1245. [CrossRef]

5. Morakul, S.; Mouret, J.R.; Nicolle, P.; Trelea, C.; Sablayrolles, J.M.; Athes, V. Modelling of the gas-liquid partitioning of aroma compounds during wine alcoholic fermentation and prediction of aroma losses. Process. Biochem. 2011, 46, 1125-1131. [CrossRef]

6. Mouret, J.R.; Sablayrolles, J.M.; Farines, V. Study and modeling of the evolution of gas-liquid partitioning of hydrogen sulfide in model solutions simulating winemaking fermentations. J. Agric. Food Chem. 2015, 63, 3271-3278. [CrossRef]

7. Aguera, E.; Sire, Y.; Mouret, J.R.; Sablayrolles, J.M.; Farines, V. Comprehensive Study of the Evolution of the Gas-Liquid Partitioning of Acetaldehyde during Wine Alcoholic Fermentation. J. Agric. Food Chem. 2018, 66, 6170-6178. [CrossRef]

8. Rollero, S.; Sanchez, I.; Camarasa, C.; Ortiz-Julien, A.; Sablayrolles, J.M.; Dequin, S. Key role of lipid management in nitrogen and aroma metabolism in an evolved wine yeast strain. Microb. Cell Fact. 2016, 15, 32. [CrossRef]

9. Colombie, S.; Latrille, E.; Sablayrolles, J.M. Interest of on-line monitoring electrical conductivity during wine fermentation. Eur. Food Res. Technol. 2008, 226, 1553-1557. [CrossRef]

10. Soulie, R. Interest of the Measurement of Dissolved $\mathrm{CO}_{2}$ during Alcoholic Fermentation in Oenological Conditions. Master's Thesis, INSA Toulouse, Toulouse, France, 1987.

11. Soubeyrand, V.; Julien, A.; Sablayrolles, J.M. Rehydration of active dry wine yeasts: Effect of the main parameters and search for early markers of yeast activity. Am. J. Enol. Vitic. 2006, 57, 474-480.

12. Moenne, I.; Mouret, J.R.; Sablayrolles, J.M.; Agosin, E.; Farines, V. Control of Bubble-free Oxygenation with Silicone Tubing during Alcoholic Fermentation. Proc. Biochem. 2013, 48, 1453-1461. [CrossRef]

13. Rivellin, N.; Barbisan, D.; Badocco, D.; Pastore, P.; Meneghesso, G.; Meneghini, M.; Cenedese, A. Study and development of a fluorescence based sensor system for monitoring oxygen in wine production: The WOW project. Sensors 2018, 18, 1130. [CrossRef]

14. Roustan, J.L.; Sablayrolles, J.M. Feasibility of measuring ferricyanide reduction by yeasts to estimate their viability during alcoholic fermentation in wine making conditions. J. Biosc. Bioeng. 2003, 96, 434-437. [CrossRef]

15. Cañete, E.; Chen, J.; Martín, C.; Rubio, B. Smart winery: A real-time monitoring system for structural health and ullage in fino style wine casks. Sensors 2018, 18, 803. [CrossRef]

16. Manginot, C.; Roustan, J.L.; Sablayrolles, J.M. Nitrogen demand of different yeast strains during alcoholic fermentation. Importance of the stationary phase. Enz. Microb. Technol. 1998, 23, 511-517. [CrossRef]

17. Julien, A.; Roustan, J.-L.; Dulau, L.; Sablayrolles, J.M. Comparison of nitrogen and oxygen demands of enological yeasts: Technological consequences. Am. J. Enol. Vitic. 2000, 51, 215-222.

18. Farines, V.; Mouret, J.R.; Morakul, S.; Moenne, I.; Sablayrolles, J.M. Development of innovative tools for the control of alcoholic fermentation. In Proceedings of the 26th World Congress of the OIV, Bucharest, Romania, 2-7 June 2013.

19. Schmidtke, L.M.; Clark, A.C.; Scollary, G.R. Micro-oxygenation of red wine: Techniques, applications, and outcomes. Crit. Rev. Food Sci. Nutr. 2011, 51, 115-131. [CrossRef]

20. Clement, T.; Perez, M.; Mouret, J.R.; Sablayrolles, J.M.; Camarasa, C. Use of a continuous multistage bioreactor to mimic winemaking fermentation. Int. J. Food Microb. 2011, 150, 42-49. [CrossRef]

21. Clement, T.; Perez, M.; Mouret, J.R.; Sanchez, I.; Sablayrolles, J.M.; Camarasa, C. Metabolic responses of Saccharomyces cerevisiae to valine and ammonium pulses during four-stage continuous wine fermentations. Appl. Environ. Microb. 2013, 79, 2749-2758. [CrossRef]

22. Casenave, C.; Perez, M.; Dochain, D.; Harmand, J.; Rapaport, A.; Sablayrolles, J.M. Anti-windup input-output linearization strategy for the control of a multi-stage continuous fermenter with input constraints. IEEE T. Contr. Syst. T. 2020, 28, 766-775. [CrossRef]

23. Colombie, S.; Malherbe, S.; Sablayrolles, J.M. Modeling of heat transfer in tanks during wine-making fermentation. Food Control 2007, 18, 953-960. [CrossRef]

24. Goelzer, A.; Charnomordic, B.; Colombie, S.; Fromion, V.; Sablayrolles, J.M. Simulation and optimization software for alcoholic fermentation in winemaking conditions. Food Control 2009, 20, 635-642. [CrossRef]

25. Plouy, D. Characterisation of Winemaking Fermentors at the Pilot Scale. Master's Thesis, Fachhochschule Giessen-Friedberg, Giessen, Germany, 2000.

26. Casalta, E.; Aguera, E.; Picou, C.; Rodriguez-Bencomo, J.J.; Salmon, J.M.; Sablayrolles, J.M. A comparison of laboratory and pilot-scale fermentations in winemaking conditions. Appl. Microb. Biotechnol. 2010, 87, 1665-1673. [CrossRef] [PubMed]

27. Malherbe, S. Modelling of Alcoholic Fermentation in Winemaking Conditions. Ph.D. Thesis, University of Montpellier II, Monpellier, France, 2003.

28. Dambergs, R.G.; Sparrow, A. The "Bodum French Press": A simple, reliable, small-lot fermentation method. In Proceedings of the 14th Australian Wine Industry Technical Conference, Adelaide, Australia, 3-8 July 2010. 
29. Aguera, E.; Sablayrolles, J.M. Pilot Scale Vinifications (1001). Wine Internet Journal n ${ }^{\circ}$ 5. 2005. Available online: www.vinidea.net (accessed on 7 July 2005).

30. Schmid, F.; Schad, J.; Jiranek, V.; Block, D.E. Formation of temperature gradients in large-and small-scale red wine fermentations during cap management. Aust. J. Grape Wine Res. 2009, 15, 249-255. [CrossRef]

31. Sparrow, A.M.; Smart, R.E. Fermentation volume studies for red wine experimentation. S. Afr. J. Enol. Viticult. 2015, 36, 343-346. [CrossRef]

32. Sampaio, T.L.; Kennedy, J.A.; Vasconcelos, M.C. Use of Microscale Fermentations in Grape and Wine Research. Am. J. Enol. Vitic. 2007, 58, 534-539.

33. Lopes, C.A.; Van Broock, M.; Querol, A.; Caballero, A.C. Saccharomyces cerevisiae wine yeast populations in a cold region in Argentinean Patagonia. A study at different fermentation scales. J. Appl. Microbiol. 2002, 93, 608-615. [CrossRef]

34. Tufariello, M.; Capozzi, V.; Spano, G.; Cantele, G.; Venerito, P.; Mita, G.; Grieco, F. Effect of co-inoculation of Candida zemplinina, Saccharomyces cerevisiae and Lactobacillus plantarum for the industrial production of Negroamaro wine in Apulia (Southern Italy). Microorganisms 2020, 8, 726. [CrossRef]

35. Cadiere, A.; Aguera, E.; Caille, S.; Ortiz-Julien, A.; Dequin, S. Pilot-scale evaluation the enological traits of a novel, aromatic wine yeast strain obtained by adaptive evolution. Food Microbiol. 2012, 32, 332-337. [CrossRef]

36. Bely, M.; Sablayrolles, J.M.; Barre, P. Automatic detection of assimilable nitrogen deficiencies during alcoholic fermentation in enological conditions. J. Ferm. Bioeng. 1990, 70, 246-252. [CrossRef]

37. Seguinot, P.; Rollero, S.; Sanchez, I.; Sablayrolles, J.M.; Ortiz-Julien, A.; Camarasa, C.; Mouret, J.R. Impact of the timing and the nature of nitrogen additions on the production kinetics of fermentative aromas by Saccharomyces cerevisiae during winemaking fermentation in synthetic media. Food Microbiol. 2018, 76, 29-39. [CrossRef]

38. Gobert, A.; Tourdot-Maréchal, R.; Sparrow, C.; Morge, C.; Alexandre, H. Influence of nitrogen status in wine alcoholic fermentation. Food Microbiol. 2019, 83, 71-85. [CrossRef]

39. Blateyron, L.; Sablayrolles, J.M. Stuck and slow fermentations in enology: Statistical study of causes and effectiveness of combined additions of oxygen and diammonium phosphate. J. Biosc. Bioeng. 2001, 91, 184-189. [CrossRef]

40. Ochando, T.; Mouret, J.R.; Humbert-Goffard, A.; Sablayrolles, J.M.; Farines, V. Impact of initial lipid content and oxygen supply on alcoholic fermentation in Champagne-like musts. Food Res. Int. 2017, 98, 87-94. [CrossRef]

41. Casalta, E.; Vernhet, A.; Sablayrolles, J.M.; Tesniesre, C.; Salmon, J.M. Characterization and role of grape solids during alcoholic fermentation under enological conditions. Am. J. Enol. Vitic. 2016, 67, 133-138. [CrossRef]

42. Casalta, E.; Salmon, J.M.; Picou, C.; Sablayrolles, J.M. Grape solids: Lipid composition and role during alcoholic fermentation under oenological conditions. Am. J. Enol. Vitic. 2019, 70, 147-154. [CrossRef]

43. Sablayrolles, J.M. Wine yeast nutrients in alcoholic fermentation: Role and management to improve alcoholic fermentation kinetics. In Proceedings of the 2nd International Wine Nutrition School, Bologne, Italy, 16 May 2019.

44. Sablayrolles, J.M.; Barre, P. Automatic control of the fermentation temperature under oenological conditions. Sci. Aliments 1989, 9, 239-251.

45. Rollero, S.; Bloem, A.; Camarasa, C.; Sanchez, I.; Ortiz-Julien, A.; Sablayrolles, J.M.; Dequin, S.; Mouret, J.R. Combined effects of nutrients and temperature on the production of fermentative aromas by Saccharomyces cerevisiae during wine fermentation. Appl. Microb. Biotech. 2019, 99, 2291-2304. [CrossRef]

46. Molina, A.M.; Swiegers, J.H.; Varela, C.; Pretorius, I.S.; Agosin, E. Influence of wine fermentation temperature on the synthesis of yeast-derived volatile aroma compounds. Appl. Microbiol. Biotechnol. 2007, 77, 675-687. [CrossRef]

47. Malherbe, S.; Fromion, V.; Hilgert, N.; Sablayrolles, J.M. Modeling the effects of assimilable nitrogen and temperature on fermentation kinetics in enological conditions. Biotech. Bioeng. 2004, 86, 261-272. [CrossRef]

48. Mouret, J.R.; Farines, V.; Sablayrolles, J.M.; Trelea, C. Prediction of the production kinetics of the main fermentative aromas in winemaking fermentations. Biochem. Eng. J. 2015, 103, 211-218. [CrossRef]

49. Aceves, C.; Athès, V.; Buche, P.; Della Valle, G.; Farines, V.; Fonseca, F.; Guillard, V.; Kansou, K.; Kristiawan, M.; Monclus, V.; et al. The virtual food system: Innovative models and experience feedback in the technologies of winemaking, cereals chain, packaging and eco-design of starter production. Innov. Food Sci. Emerg. Technol. 2018, 46, 54-64. [CrossRef]

50. Mouret, J.R.; Farines, V.; Athès, V.; Aguera, E.; Lutton, E.; Boukhelifa, N.; Bideaux, C.; Aceves Lara, C.A.; Trelea ICAnd Sablayrolles, J.M. Winemaking fermentation: From online monitoring to modeling and multicriteria optimization. In Proceedings of the 9th Specialized Conference on Sustainable Viticulture, Winery Wastes \& Agro-industrial Wastewater Management, Mons, Belgium, 3-5 June 2019.

51. Neveu, P.; Rossard, V.; Tireau, A.; Aguera, E.; Perez, M.; Picou, C.; Sablayrolles, J.M. Software for data and knowledge management in winemaking fermentations. In Proceedings of the International Conference on Knowledge Engineering and Ontology Development (KEOD-2012), Barcelona, Spain, 4-7 October 2012.

52. Sablayrolles, J.M. Kinetics and control of alcoholic fermentation during wine production. In Yeasts in the Production of Wine; Romano, P., Ciani, M., Fleet, H., Eds.; Springer: New York, NY, USA, 2019; pp. 283-313.

53. Morata, A.; Escott, C.; Loira, I.; Del Fresno, J.M.; González, C.; Suárez-Lepe, J.A. Influence of Saccharomyces and NonSaccharomyces Yeasts in the Formation of Pyranoanthocyanins and Polymeric Pigments during Red Wine Making. Molecules 2019, 24, 4490. [CrossRef] 
54. Tufariello, M.; Fragasso, M.; Pico, J.; Panighel, A.; Castellarin, S.D.; Flamini, R.; Grieco, F. Influence of non-Saccharomyces on wine chemistry: A focus on aroma-related compounds. Molecules 2021, 26, 644. [CrossRef]

55. Del Fresno, J.M.; Morata, A.; Loira, I.; Bañuelos, M.A.; Escott, C.; Benito, S.; Chamorro Cg Suárez-Lepe, J.A. Use of nonSaccharomyces in single-culture, mixed and sequential fermentation to improve red wine quality. Eur. Food Res. Technol. 2017, 243, 2175-2185. [CrossRef]

56. Ciani, M.; Capece, A.; Comitini, F.; Canonico, L.; Siesto, G.; Romano, P. Yeast Interactions in Inoculated Wine Fermentation. Front. Microbiol. 2016, 7, 555. [CrossRef]

57. Pommier, S.; Albasi, C.; Riba, J.P.; Delia, M.L. A new membrane tool for quantifying microorganism interaction dynamics. Application to yeast killer systems. Desalination 2002, 149, 243-245. [CrossRef]

58. Renault, P.E.; Albertin, W.; Bely, M. An innovative tool reveals interaction mechanisms among yeast populations under oenological conditions. Appl. Microbiol. Biotechnol. 2013, 97, 4105-4119. [CrossRef]

59. Ogawa, M.; Bisson, L.F.; Garcia-Martinez, T.; Mauricio, J.C.; Moreno-Garcia, J. New insights on yeast and filamentous fungus adhesion in a natural co-immobilization system: Proposed advances and applications in wine industry. Appl. Microbiol. Biotechnol. 2019, 103, 4723-4731. [CrossRef]

60. Terpou, A.; Ganatsios, V.; Kanellaki, M.; Koutinas, A.A. Entrapped psychrotolerant yeast cells within pine sawdust for low temperature wine making: Impact on wine quality. Microorganisms 2020, 8, 764. [CrossRef]

61. Moreno-Garcia, J.; Garcia-Martinez, T.; Mauricio, J.C.; Moreno, J. Yeast Immobilization Systems for Alcoholic Wine Fermentations: Actual Trends and Future Perspectives. Front. Microbiol. 2018, 9, 241. [CrossRef] [PubMed]

62. Bleve, G.; Tufariello, M.; Vetrano, C.; Mita, G.; Grieco, F. Simultaneous alcoholic and malolactic fermentations by Saccharomyces cerevisiae and Oenococcus oeni cells co-immobilized in alginate beads. Front. Microbiol. 2016, 7, 943. [CrossRef] [PubMed]

63. Cañete-Carmona, E.; Gallego-Martínez, J.J.; Martín, C.; Brox, M.; Luna-Rodríguez, J.J.; Moreno, J. A Low-Cost IoT Device to Monitor in Real-Time Wine Alcoholic Fermentation Evolution Through $\mathrm{CO}_{2}$ Emissions. IEEE Sens. J. 2020, 20, 6692-6700. [CrossRef]

64. Opitz, C.; Schade, G.; Kaufmann, S.; Di Berardino, M.; Ottiger, M.; Grzesiek, S. Rapid determination of general cell status, cell viability, and optimal harvest time in eukaryotic cell cultures by impedance flow cytometry. Appl. Microbiol. Biotechnol. 2019, 103, 8619-8629. [CrossRef]

65. Vees, C.C.; Veiter, L.; Sax, F.; Herwig, C.; Pfluegl, S. A robust flow cytometry-based biomass monitoring tool enables rapid at-line characterization of S. cerevisiae physiology during continuous bioprocessing of spent sulfite liquor. Anal. Bioanal. Chem. 2020, 412, 2137-2149. [CrossRef]

66. Jimenez-Marquez, F.; Vazquez, J.; Sanchez-Rojas, J.L. Optoelectronic sensor device for monitoring the maceration of red wine: Design issues and validation. Measurement 2015, 63, 128-136. [CrossRef]

67. Shrake, N.L.; Amirtharajah, R.; Brenneman, C.; Boulton, R.; Knoesen, A. In-Line Measurement of Color and Total Phenolics during Red Wine Fermentations Using a Light-Emitting Diode Sensor. Am. J. Enol. Vitic. 2014, 65, 463-470. [CrossRef]

68. Vasilescu, A.; Fanjul-Bolado, P.; Titoiu, A.M.; Porumb, R.; Epure, P. Progress in Electrochemical (Bio)Sensors for Monitoring Wine Production. Chemosensors 2019, 7, 66. [CrossRef]

69. Buratti, S.; Benedetti, S. Alcoholic Fermentation Using Electronic Nose and Electronic Tongue). In Electronic Noses and Tongues in Food Science; Mendez, M.L.R., Ed.; Academic Press LTD-Elsevier Science: London, UK, 2016; pp. 291-299.

70. Jiang, H.; Zhang, H.; Chen, Q.S.; Mei, C.L.; Liu, G.H. Recent advances in electronic nose techniques for monitoring of fermentation process. World J. Microbiol. Biotechnol. 2015, 31, 1845-1852. [CrossRef]

71. Liu, H.; Li, Q.; Yan, B.; Zhang, L.; Gu, Y. Bionic Electronic Nose Based on MOS Sensors Array and Machine Learning Algorithms Used for Wine Properties Detection. Sensors 2019, 19, 45. [CrossRef]

72. Berbegal, C.; Khomenko, I.; Russo, P.; Spano, G.; Fragasso, M.; Biasioli, F.; Capozzi, V. PTR-ToF-MS for the online monitoring of alcoholic fermentation in wine: Assessment of VOCs variability associated with different combinations of Saccharomyces/nonSaccharomyces as a case-study. Fermentation 2020, 6, 55. [CrossRef]

73. Puxeu, M.; Andorra, I.; Lamo-Castellví, D.; Ferrer-Gallego, R. Determination of Nutrient Supplementation by Means of ATR-FTIR Spectroscopy during Wine Fermentation. Fermentation 2019, 5, 58. [CrossRef]

74. Simonin, S.; Roullier-Gall, C.; Ballester, J.; Schmitt-Kopplin, P.; Quintanilla Casas, B.; Vichi, S.; Alexandre, H.; Tourdot-Marechal, R. Bio-protection as an alternative to sulphites: Impact of chemical and microbiological characteristics on red wines. Front. Microbiol. 2020, 11, 1308. [CrossRef] 